\title{
Uninterrupted Coverage of a Planar Region with Rotating Directional Antennae
}

\author{
Evangelos Kranakis ${ }^{1}$, Fraser MacQuarie $^{2}$, Oscar Morales-Ponce ${ }^{3}$, and Jorge Urrutia ${ }^{4}$ \\ 1 School of Computer Science, Carleton University, Ottawa, Canada \\ Supported in part by NSERC and MITACS grants. kranakisescs. carleton.ca \\ 2 School of Computer Science, Carleton University, Ottawa, Canada \\ frasermacquarrie@gmail.com \\ 3 School of Computer Science, Carleton University, Ottawa, Canada \\ Supported by MITACS Postdoctoral Fellowship. omponce@ connect. carleton.ca \\ 4 Instituto de Matemáticas, Universidad Nacional Autónoma de México, México \\ Supported in part by Conacyt. urrutia@matem. unam.mx
}

\begin{abstract}
Assume that $n$ directional antennae located at distinct points in the plane are rotating at constant identical speeds. They all have identical range and sensor angle (or field of view). We propose and study the Rotating Antennae Coverage Problem, a new problem concerning rotating sensors for the uninterrupted coverage of a region in the plane. More specifically, what is the initial orientation of the sensors, minimum angle, and range required so that a given (infinite or finite) line or planar domain is covered by the rotating sensors at all times? We give algorithms for determining the initial orientation of the sensors and analyze the resulting angle/range tradeoffs for ensuring continuous coverage of a given region or line in the plane with identical rotating sensors of given transmission angle and range. We also investigate other variants of the problem whereby for a given parameter $T$ (representing time) there is no point in the domain that is left unattended by some sensor for a period of time longer than $T$. Despite the apparent simplicity of the problem several of the algorithms proposed are intricate and elegant. We have also implemented our algorithms in $\mathrm{C}++$ and the code can be downloaded on the web.
\end{abstract}

Key Words and Phrases: Angle, Antenna, Constant Speed, Coverage, Floodlights, Rotating, Sensors.

\section{Introduction}

Assume $n$ directional antennae with identical range and beam width and located at distinct points in a planar finite or infinite domain. The antennae are rotating continuously at constant identical speeds. A point in the domain is called covered by a sensor if it is within the range and coverage area of at least one of the $n$ sensors. The domain may well represent a critical region all of whose points need to be covered so as to monitor important events (such as animal migration, military activity, navigation guidance, etc.) which is taking place within this domain. In this setting it is required that specific events that may occur at some point within this domain be detected, located and reported by 
at least one of the sensors at all times. More specifically we consider the following Rotating Antennae Coverage Problem concerning the monitoring of a region.

Assume we are given a finite or infinite planar region. We have $n$ sensors modelled as directional antennae with given identical ranges and beam widths. The sensors are rotating continuously with constant identical speeds. We are concerned with providing an algorithm for determining the initial orientation of the antennae so as to ensure that no point in the domain is ever left unmonitored at any time. In addition, we are also interested in algorithms for attaining optimal antennae angle/range tradeoffs for accomplishing this monitoring task.

In a further (and natural) generalization, we may also be interested in two additional parameters. 1) Gap Time $T$ : for some real number $T \geq 0$, it is required that specific events that may occur at some point in this domain be detected, located and reported by at least one sensor within any specified time interval whose length does not exceed a certain gap $T$, and 2) Number of Monitoring Antennae $k$ : for some integer $k \geq 1$, every point in the region is monitored by at least $k$ antennae at all times. We use the notation $R A C_{k}(T)$ to denote this Rotating Antennae Coverage problem with monitoring time $T$ and number of monitors $k$. When $k=1$ we use the abbreviation $R A C(T)$, when $T=0$ the abbreviation $R A C_{k}$, and when both $k=1, T=0$ we simply use the abbreviation $R A C$. In particular, in $R A C_{k}$ we want to ensure that every point in the region is always monitored by at least $k$ sensors at all times. Thus, despite the fact that the coverage provided by each individual sensor may be intermittent (due to limitations on the antenna angle and range) and may result in insufficiently covered "corridors" within the plane region during the antenna rotation, the coverage provided by the ensemble of all the rotating sensors when taken together guarantees complete coverage of the region at all times.

To address the Rotating Antennae Coverage problem we propose a rotation model whereby directional antennae rotate at constant identical speeds in the same direction. This same model could also be used if it was required to locate the activities and report events if sensors were also location aware (i.e., they knew their geographic coordinates).

\subsection{Preliminaries, definitions, and notation}

In the sequel we define our coverage problem precisely and provide basic terminology, definitions and notation. Throughout the paper we assume that we have $n$ identical directional sensors. Each sensor consists of a rotating directional antenna with range (also called radius) $r>0$, beam width (also called angle) $0 \leq \phi \leq 2 \pi$ that rotates around its apex (which is at a fixed position) with constant speed in clockwise order. All antennae rotate in the same direction at constant identical speeds. The antennae are set at some initial orientation (determined by an algorithm) that depends on the particular location of its sensor in relation to the remaining sensors in the set of points. The coverage area of a sensor at time $t$ is the circular sector of radius $r$ and angle $\phi$ determined by the sensor during its rotation at time $t$. A point in a given planar region $\mathcal{R}$, is called covered at time $t$ if it is within the range of at least one of the $n$ sensors at time $t$. We study the problem of covering $\mathcal{R}$ with a set of rotating directional sensors of identical angle $\phi$ 
and range $r$. We distinguish two types of sensors: 1) directional sensors with given angle and finite range, for example, video cameras, and 2) directional sensors with given angle but unlimited (or infinite) range, which we refer to in the sequel as floodlights.

Note that although floodlights (i.e., sensors with infinite range) may not be technically realistic, nevertheless they will prove to be quite convenient in subsequent discussions in that they will simplify proofs and mathematical presentation. With these explanations in mind we are ready to give the main definitions.

Definition 1 (Angles $\Phi_{r}(P, \mathcal{R})$ and $\Phi(P, \mathcal{R})$ ). Let $P$ be a set of points in the plane and $\mathcal{R}$ be a planar region. Let $\Phi_{r}(P, \mathcal{R})$ be the infimum over all angles $\phi \leq 2 \pi$ such that if sensors of angle $\phi$ and range $r$ are located at the points then there is an initial orientation of the sensors so that the whole region $\mathcal{R}$ is covered at all times under continuous rotation of the directional antennae. For the case of floodlights we have infinite range $r=+\infty$ in which case we use the notation $\Phi(P, \mathcal{R})$.

Definition 2 (Angles $\Phi_{r}(n, \mathcal{R})$ and $\Phi(n, \mathcal{R})$ ). Let $\mathcal{R}$ be a region in the plane. Let $\Phi_{r}(n, \mathcal{R})$ be the infimum over all $\Phi_{r}(P, \mathcal{R})$ where $P$ is any set of $n$ directional sensors in the plane. For the case of floodlights we have infinite range $r=+\infty$ in which case we use the notation $\Phi(n, \mathcal{R})$.

We note that although in the sequel we will be assuming that the sensors lie in the region $\mathcal{R}$ under consideration the definitions make sense even without this assumption. A similar definition can be given for covering a line $\mathcal{L}$ (i.e., only for points located on the line) using rotating antennae and the corresponding notation is $\Phi(n, \mathcal{L})$. The coverage problems we are interested in can be formulated precisely as follows.

Problem 1. Determine the beam width $\Phi_{r}(P, \mathcal{R})$ such that there is an initial orientation of the sensors in $P$ with range $r$ so that the whole region $\mathcal{R}$ is covered under continuous rotation of the directional sensors. Similar problem for $\Phi(P, \mathcal{L})$.

Problem 2. Determine the beam width $\Phi_{r}(n, \mathcal{R})$ such that there is an initial orientation of $n$ directional sensors with range $r$ so that the whole region $\mathcal{R}$ is covered under continuous rotation of the directional sensors. Similar problem for $\Phi(n, \mathcal{L})$.

We will see in the sequel that the coverage problems for infinite and finite range are related. As usual, $\angle(A B C)$ denotes the angle between the line segments $A B$ and $B C$. Assume we have a point $K=(x, y)$. For any angle $\rho$ define the point $K_{\rho}=(x, y)+r e^{i \rho}$.

Definition 3 (Rotating Antenna Sector). Consider a directional antenna located at a point $K$ with beam width $\phi$ and radius $r$ as it rotates clockwise. We define as follows the sector delimited by the antenna at time $t$.

-Let $F_{K}(r, \rho ; 0)$ denote the initial sector defined by the sensor when its orientation is $\rho$; this is the circular sector defined in a circle of radius $r$, centered at $K$ and delimited by the radii $K K_{\rho}$ and $K K_{\rho+\phi}$.

-At time $t$ the sensor will rotate by an angle of $t$ radians. Let $F_{K}(r, \rho ; t)$ denote the circular sector at time $t$ which is defined in a circle of radius $r$, centered at $K$ and delimited by the radii $K K_{\rho-t}$ and $K K_{\rho-t+\phi}$. 
Although we omit the details, a similar definition can be given when $r$ is infinite and we simply denote the sector defined by the sensor located at the point $K$ by $F_{K}(\rho ; t)$. Observe that the orientation at time $t$ is invariant to the initial orientation, i.e., $F_{K}(r, \rho ; t)=F_{K}(r, \rho-t ; 0)$.

\subsection{Related work}

There exists research in computational geometry that is somewhat related to our problem. For example, the art gallery problem which is concerned with placing the minimum number of guards in a planar domain so as to cover a given region or perimeter and has been studied in various different settings. For the art gallery problem, Chvatal [2] proved that $n / 3$ guards are always sufficient and sometimes necessary to guard a simple polygon with $n$ vertices and later Fisk [3] gave a shorter proof. In these works, guards have an omnidirectional field of view. For additional details on art gallery problems the reader is referred to [7,9], as well as to [4] for a more recent randomized algorithm for sensor placement in a simple polygon. Closely related is research with floodlights which corresponds to our antenna model with fixed angle but infinite range. For example, [11] proposes the problem of illuminating the plane with floodlights and proves that the infinite plane can be illuminated with $n$ floodlights if and only if the sum of angles is at least $2 \pi$.

There is extensive literature in mobile and sensor networks concerning coverage, e.g., see $[10,1]$. The $k$-coverage problem with isotropic sensors was studied in [6]. In [12] and [5] the authors studied the $k$-coverage problem and the relationship between coverage and connectivity. Additional research can also be found in [8].

It is important to point out that all the literature mentioned above differs from our setting in that the antennae are static while we are concerned with a dynamic model of rotating antennae.

\subsection{Results of the Paper}

We provide several algorithms depending on the number of points and their relative location that determine for a given set of points in the plane the initial orientation of the sensors, as well as minimum angle, and range required so that a given (infinite or finite) line or planar domain is covered at all times regardless of the fact that the sensors are rotating. We give algorithms for determining the initial orientation of the sensors and study angle/range tradeoffs given that the sensors rotate with identical speeds and have a given field of view and range. Section 2 is concerned with lattice configurations, and Section 3 with arbitrary configurations of points in the plane. In both cases we consider algorithms for orienting the antennae so as to cover a given line or region provided the sensors are located in lattice configurations or arbitrary positions in the plane. In Section 4 we look at other variants of the problem for a given parameter $T$ (representing the gap time) whereby no point in the domain is left unattended by a sensor for a period longer than $T$. Several of the algorithms proposed are intricate and elegant. We conclude with discussion of open problems. The main results of the paper are summarized in Table 1, for infinite, and Table 2 for finite antennae, respectively. 


\begin{tabular}{|l|c|c|}
\hline Points $P$ in & Range & Beam Width \\
\hline Line $\mathcal{L}$ of size $r$ & $r$ & $\Phi_{r}(P, \mathcal{L})=\frac{3 \pi}{n}$ \\
Lattice $\mathcal{L}$ of size $m \times n$ & $r \leq 2 \max (n, m) / 3$ & $\Phi_{r}(P, \mathcal{L}) \geq \frac{2 \pi}{r}$ \\
Lattice $\mathcal{L}$ of size $m \times n$ & $r \leq 2 \max (n, m) / 3$ & $\Phi_{r}(P, C H(\mathcal{L})) \geq \frac{2 \pi}{\sqrt{r^{2}-1}}$ \\
General Position & $r_{D T(P)}$ & $\Phi_{r}(P, C H(P)) \geq \pi$ \\
\hline
\end{tabular}

Table 1: Summary of results with infinite range. We use the notation $r_{D T(P)}=$ $2 \max _{u, v}(d(u, v):\{u, v\} \in D T(P))$, where $D T(P)$ is the Delaunay Triangulation of the set of points.

\begin{tabular}{|l|c|c|}
\hline Points $P$ in & Coverage Region & Beam Width \\
\hline Line $\mathcal{L}$ & $\mathcal{L}$ & $\Phi(P, \mathcal{L})=\frac{3 \pi}{n}$ \\
Line $\mathcal{L}$ & $\mathcal{H}_{u}(\mathcal{L})$ & $\Phi\left(P, \mathcal{H}_{u}(\mathcal{L})\right)=\frac{3 \pi}{n}$ \\
General Position & Plane $\mathcal{P}$ & $\Phi(2, \mathcal{P})=2 \pi$ \\
General Position & Plane $\mathcal{P}$ & $\Phi(3, \mathcal{P})=\pi$ \\
\hline
\end{tabular}

Table 2: Summary of results with finite range. We use the notation $\mathcal{H}_{u}(\mathcal{L})$ to denote the upper half-plane determined by $\mathcal{L}$.

\section{Lattice Configurations}

In this section we consider sensors located in lattice positions, namely the $1 \times n$ and $m \times n$ grid.

\subsection{Infinite line}

Theorem 1. For any set $P$ of $n \geq 2$ floodlights on a line $\mathcal{L}$ we have that $\Phi(P, \mathcal{L})=\frac{3 \pi}{n}$.

Proof. Without loss of generality assume that the line $\mathcal{L}$ to be covered is horizontal. Let $P=\left\{p_{0}, p_{1}, \ldots, p_{n-1}\right\}$ be the set of $n$ sensors on the line $\mathcal{L}$ and let the points be such that the $x$-coordinate of $p_{i}$ is less than the $x$-coordinate of $p_{i+1}$, for $i=0,1, \ldots, n-2$.

First we prove that an angle of $\frac{3 \pi}{n}$ is always sufficient. Let the initial orientation of the sensor at $p_{i}$ be $F_{p_{i}}(i \cdot 3 \pi / n ; 0)$, for $i=0,1, \ldots, n-1$; see Figure 1 . We define the

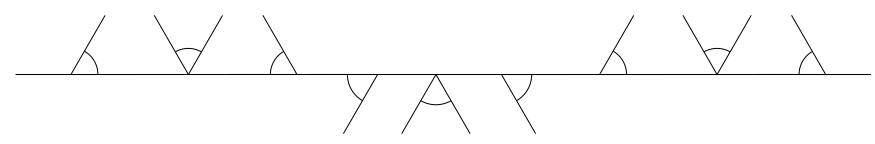

Fig. 1: Initial orientation of the directional sensors on $\mathcal{L}$.

dual plane as follows: each sensor $i$ is the circular sector of a unitary circle $C$ delimited by $i \cdot 3 \pi / n$ and $(i+1) \cdot 3 \pi / n$, and at time $t$, the line $\mathcal{L}$ is represented as a directed line 
segment $\vec{L}$ such that $\overrightarrow{\mathcal{L}}$ crosses the center of $C$ and the head of $\overrightarrow{\mathcal{L}}$ forms an angle $t$ with the horizontal; see Figure $2 b$.

In the dual plane, sensors are static while it is the line $\mathcal{L}$ that rotates all the time. The orientation $\overrightarrow{\mathcal{L}}$ of $\mathcal{L}$ preserves the sensor rotations in the original plane. The head of $\overrightarrow{\mathcal{L}}$ represents $\infty$ and the tail represents $-\infty$ in the original plane.

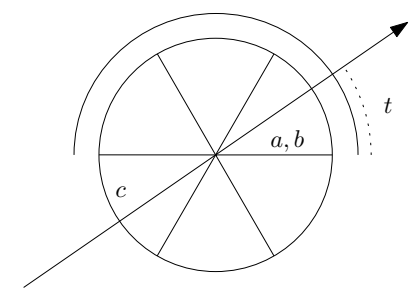

(a) Orientation at $t$.

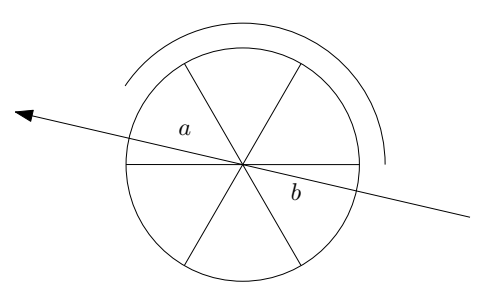

(b) If $\Phi(n, \mathcal{L})<\frac{3 \pi}{n}, \mathcal{L}$ is not always fully covered.

Fig. 2: Directional sensors at a unique point.

Since the sum of the angles is $3 \pi$, the circular sector $[0, \pi)$ of $C$ in the dual plane is always covered by two sets $S_{1}, S_{2} \subseteq P$ of sensors while the circular sector $[\pi, 2 \pi)$ of $C$ in the dual plane is covered by one set $S_{3} \subseteq P$ of sensors. Observe that each sensor in $S_{3}$ is between $S_{1}$ and $S_{2}$ in the original plane. Let $a \in S_{1}, b \in S_{2}$ and $c \in S_{3}$ be the sensors that cover a segment of $\vec{L}$ at time $t$ in the dual plane. If $a$ and $b$ cover the head of $\vec{L}, c$ covers the tail. Therefore, $\mathcal{L}$ is fully covered by $c$ and $b$ in the original plane. Similarly, if $a$ and $b$ cover the tail of $\overrightarrow{\mathcal{L}}, c$ covers the head. Therefore, $\mathcal{L}$ is fully covered by $a$ and $c$ in the original plane.

Now we prove that an angle of $\frac{3 \pi}{n}$ is always necessary. Assume on the contrary that the sum of angles is less than $3 \pi$. Therefore, there exists a time $t$ when only two sensors, say $a$ and $b$, cover a segment of $\vec{L}$ in the dual plane as depicted in Figure $2 b$. Assume $a$ covers the tail and $b$ covers the head of $\overrightarrow{\mathcal{L}}$ in the dual plane. Therefore, $\mathcal{L}$ is fully covered in the original plane. However, at time $t+\pi, a$ covers the head and $b$ covers the tail of $\overrightarrow{\mathcal{L}}$ in the dual plane. Therefore, the line segment $a b$ of $\mathcal{L}$ in the original plane is not covered. This contradicts the assumption. The pseudocode is presented in Algorithm ??.

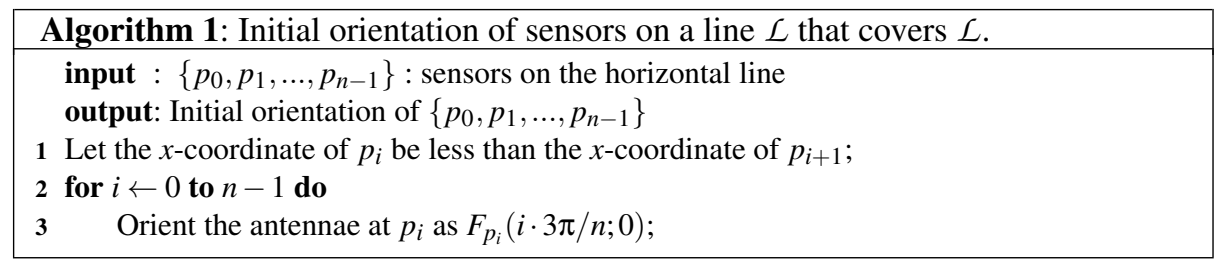


This completes the proof of the theorem.

Observe that if $\mathcal{L}$ is finite, then it is sufficient to use a range equal to the length of $\mathcal{L}$. Thus, we have the following corollary to Theorem 1 when $\mathcal{L}$ is finite.

Corollary 1. For a set $P$ of $n \geq 2$ sensors on a line $\mathcal{L}$ of length $r$, we have that $\Phi_{r}(P, \mathcal{L})=\frac{3 \pi}{n}$.

\subsection{Square lattice}

Theorem 2. Consider a set $P$ of $n$ directional sensors located in a lattice $\mathcal{L}$ of size $m \times n$ and let the antennae have range $r$ such that $\max (n, m) \geq\lceil 3 r / 2\rceil$. Then, we have that $\Phi_{r}(P, \mathcal{L}) \geq \frac{2 \pi}{r}$.

Proof. Assume without loss of generality that $n \geq m$. It is sufficient to orient the antennae and provide coverage for a single row of the lattice and apply the result to each row so as to cover each point in $P$; see Figure 3.

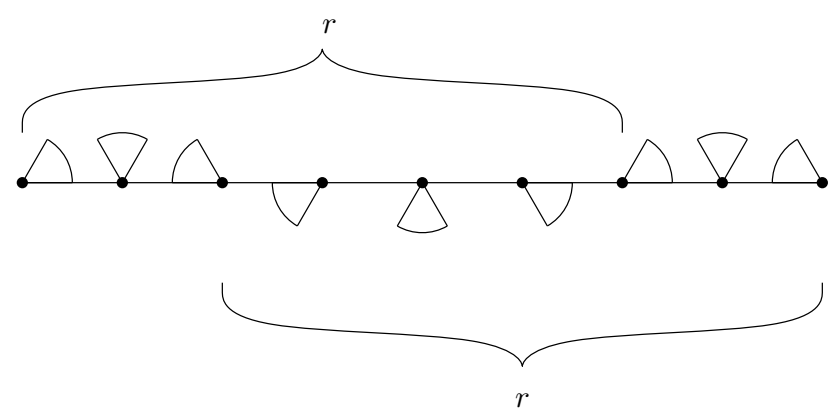

Fig. 3: $r=6$, a lattice of size $n=9$ and the initial positions.

Let $P=\left\{p_{0}, p_{1}, \ldots, p_{n-1}\right\}$ be the sensors in the row $j$ and let the points be such that the $x$-coordinate of $p_{i}$ is less than the $x$-coordinate of $p_{i+1}$. Orient the $i$ th sensor as $F_{p_{i}}(r, i \cdot \pi / r ; 0)$. To prove that $\mathcal{L}$ is covered, consider a pair of sensors $p_{i}$ and $p_{j}$ at distance $\lceil 3 r / 2\rceil$. Since $n \geq\lceil 3 r / 2\rceil$, there are $3 r / 2$ sensors between $p_{i}$ and $p_{j}$. From Corollary 1 , the line segment $p_{i} p_{j}$ is always covered since $\left\lceil\frac{3 r}{2}\right\rceil \Phi_{r}(P, \mathcal{L}) \geq 3 \pi$. The pseudocode is presented in Algorithm ??.

This completes the proof of the theorem.

\section{Planar Configurations}

In this section we consider configurations of sensors in the plane and study coverage for half-plane, infinite plane, and the convex hull of a set of points. 


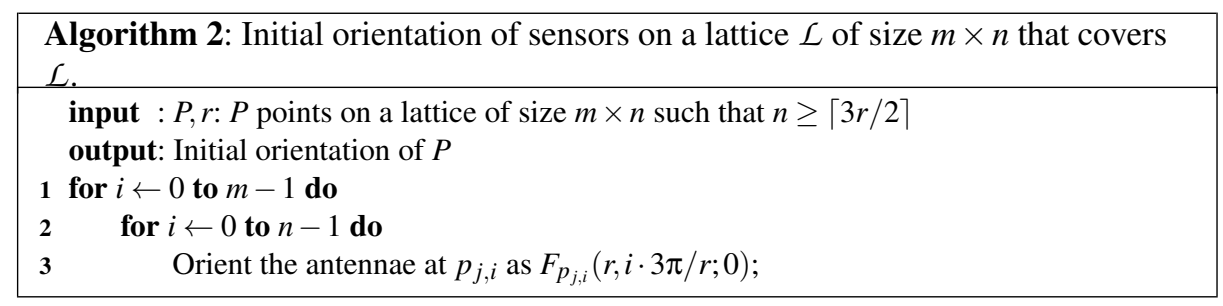

\subsection{Covering the Half-Plane}

First we consider orientation algorithms for covering a half-plane determined by an infinite line. We say that two sensors $a$ and $b$ with sensor angle $\phi$ form a dark corridor at time $t$ if $F_{a}(\rho ; t) \cap F_{b}(\rho+\phi ; t)=\emptyset$.

Lemma 1. Let $a$ and $b$ be two directional sensors of angle $\phi$ on a horizontal line. Assume that the initial orientations of the antennae at $a, b$ are $F_{a}(\pi-\phi ; 0), F_{b}(\pi ; 0)$, respectively. Further, assume that the $x$-coordinate of $a$ is less than the $x$-coordinate of b. If $0 \leq t \leq \pi$, the intersection of the sensors covers a circular sector $2 \phi$. If $\pi<t<2 \pi$, they leave a black corridor.

Proof. Let $l_{a}, l_{b}, r_{a}$ and $r_{b}$ be the left and right rays that define the wedges of the sensors at $a$ and $b$ respectively. Let $h$ be the horizontal. At time $t, \angle\left(r_{a}, h\right)=\pi-t-\phi$, $\angle\left(l_{a}, h\right)=\pi-t, \angle\left(r_{b}, h\right)=\pi-t$ and $\angle\left(l_{b}, h\right)=\pi-t+\phi$. Observe that $\angle\left(r_{a}, r_{b}\right)=-\phi$ and $\angle\left(l_{b}, l_{a}\right)=\phi$. Therefore, when $\pi<t<2 \pi$, the rays of $r_{a}$ and $l_{b}$ do not intersect, i.e., $F_{a}(\pi ; t) \cap F_{b}(\pi-\phi ; t)=\emptyset$ since the $x$-coordinate of $a$ is less than the $x$-coordinate of $b$ and a black corridor is formed; see Figure $4 \mathrm{~b}$. However, when $0 \leq t \leq \pi, r_{a}$ intersects $l_{b}$ since the $x$-coordinate of $a$ is less than the $x$-coordinate of $b$. Consider the intersection point $x$ between $r_{a}$ and $l_{b}$; see Figure $4 \mathrm{a}$. It is not difficult to see that $l_{a}$ and $l_{b}$ determine a coverage wedge incident to $x$ of angle $2 \phi$.

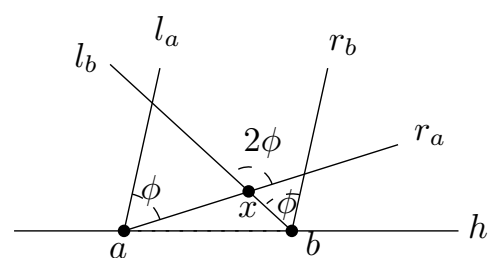

(a) $0 \leq t \leq \pi$

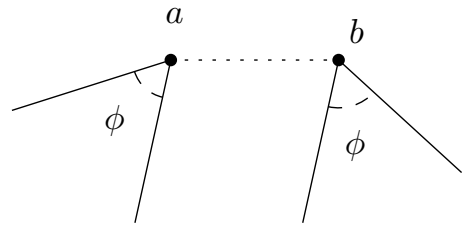

(b) $\pi<t<2 \pi$ a dark corridor is formed

Fig. 4: Two directional sensors.

Theorem 3. For a set $P$ of $n \geq 3$ floodlights on a line $\mathcal{L}$ and the upper half-plane $\mathcal{H}_{u}(\mathcal{L})$ determined by $\mathcal{L}$ we have that $\Phi\left(n, \mathcal{H}_{u}\right)=\frac{3 \pi}{n}$. 
Proof. We will prove that the initial orientation depicted in Figure 1 of Theorem 1 also covers the half-plane $\mathcal{H}_{u}(\mathcal{L})$ determined by $\mathcal{L}$. Similarly to the proof of Theorem 1 we assume that $\mathcal{L}$ is horizontal and the $x$-coordinate of the sensor $p_{i}$ is less than the $x$-coordinate of the sensor $p_{i+1}$, for all $i=0,1, \ldots, n-2$. As before, we define the dual plane as follows: 1) each sensor $i$ is the circular sector of a unitary circle $C$ delimited by $i \cdot 3 \pi / n$ and $(i+1) \cdot 3 \pi / n, 2)$ at time $t$, the line $\mathcal{L}$ is represented as a directed line segment $\overrightarrow{\mathcal{L}}$ such that $\overrightarrow{\mathcal{L}}$ crosses the center of $C$ and the head of $\overrightarrow{\mathcal{L}}$ forms an angle $t$ with the horizontal, and 3) the upper half-plane $\mathcal{H}_{u}(\mathcal{L})$ determined by $\mathcal{L}$ is represented by the left half-plane determined by $\overrightarrow{\mathcal{L}}$; see Figure $2 \mathrm{a}$.

In the dual plane sensors are static and $\mathcal{L}$ rotates during the time. The orientation $\overrightarrow{\mathcal{L}}$ of $\mathcal{L}$ preserves the sensor rotations and the upper half-plane $\mathcal{H}_{u}$ of the original plane.

Since the sum of the angles is $3 \pi$, the circular sector $[0, \pi)$ of $C$ in the dual plane is always covered twice and the circular sector $[\pi, 2 \pi)$ of $C$ is covered once. Let $S_{1}$ be the set of circular sectors that covers the head of $\overrightarrow{\mathcal{L}}$ and let $S_{2}$ be the set of circular sectors that covers the tail of $\overrightarrow{\mathcal{L}}$. Observe that either $\left|S_{1}\right|=1$ and $\left|S_{2}\right|=2$ or $\left|S_{1}\right|=2$ and $\left|S_{2}\right|=1$. We will prove that there exists an increasing subsequence $p_{j}, p_{j+1}, \ldots, p_{i}$ so as by Lemma 1 it covers the left half-space determined by $\overrightarrow{\mathcal{L}}$. If $\left|S_{1}\right|=1$, let $p_{i} \in S_{1}$ and $p_{j} \in S_{2}$ such that $j$ is the min label in $S_{2}$. Otherwise if $\left|S_{1}\right|=2$, let $p_{i} \in S_{2}$ and $p_{j} \in S_{1}$ such that $j$ is the min label in $S_{1}$. Since $j<i$, the increasing subsequence is determined by the sensors $p_{j}, p_{j+1}, \ldots, p_{i}$.

To prove the bound is tight, assume by contradiction that $\Phi\left(n, \mathcal{H}_{u}(\mathcal{L})\right)<3 \pi / n$; see Figure $2 \mathrm{~b}$. Since $\Phi\left(n, \mathcal{H}_{u}\right)<3 \pi / n$ they cover less than $3 \pi$. Therefore, there exists a time $t$ such that only two sensors fully cover $\vec{L}$. Assume $a$ covers the tail and $b$ covers the head of $\mathcal{L}$. Therefore, $\mathcal{L}$ is fully covered. However, at time $t+\pi, a$ covers the head and $b$ covers the tail of $\mathcal{L}$. Therefore, the line segment $a b$ is not covered. This contradicts the assumption. This completes the proof of the theorem.

Observe that if the points are uniformly distributed in the lower line of a rectangle of size $l \times 1$, it is sufficient a range equal to $\sqrt{l^{2}+1}$. Thus, we have a corollary to Theorem 3.

Corollary 2. For a set $P$ of $n \geq 2$ sensors uniformly distributed in the lower line of a rectangle $\mathcal{R}$ of size $l \times 1$, we have that $\Phi_{r}(P, \mathcal{R})=\frac{3 \pi}{n}$; where $r=\sqrt{l^{2}+1}$.

Theorem 4. Assume we are given a set $P$ of mn directional sensors of radius $r$ located in a $m \times n$ lattice $\mathcal{L}$ where $\max (n, m) \geq\lceil 3 r / 2\rceil$. Let $C H(\mathcal{L})$ be the convex hull of $\mathcal{L}$. Then, we have that $\Phi_{r}(P, C H(\mathcal{L})) \geq \frac{2 \pi}{\sqrt{r^{2}-1}}$.

Proof. Without loss of generality assume that $n>m$. Let $p_{i, j}$ be the sensor at row $i$ and column $j$ for $0 \leq i<m$ and $0 \leq j<n$. Orient $p_{i, j}$ as $F_{p_{i, j}}\left(r, j \cdot \frac{\pi}{\sqrt{r^{2}-1}} ; 0\right)$; see Figure ??. To prove that it is always sufficient, consider a pair of sensors $p_{i, j}$ and $p_{i, k}$ in the row $i$ at distance $\lceil 3 r / 2\rceil$. Since $n \geq\lceil 3 r / 2\rceil$, there are $3 r / 2$ sensors between $p_{i}$ and $p_{j}$. From Corollary 2 the area between row $i$ and $i+1$ is fully covered with range $r$ since $\left\lceil\frac{3 r}{2}\right\rceil \Phi_{r}(P, C H(\mathcal{L}))>3 \pi$. We give below the pseudocode of the main algorithm. This completes the proof of the theorem. 


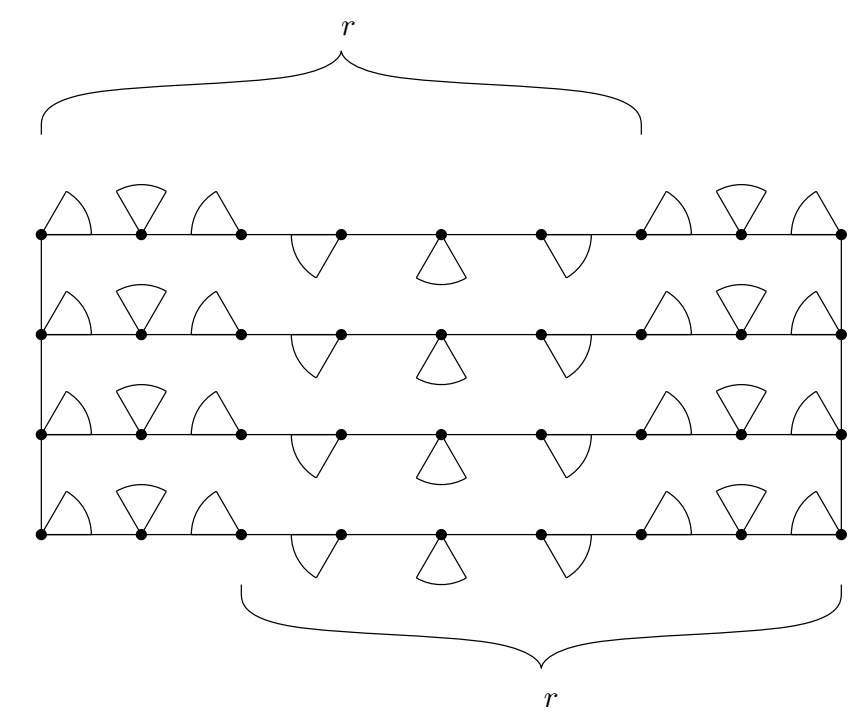

Fig. 5: $r=4$, a grid of size $4 \times 9$ and the initial position.

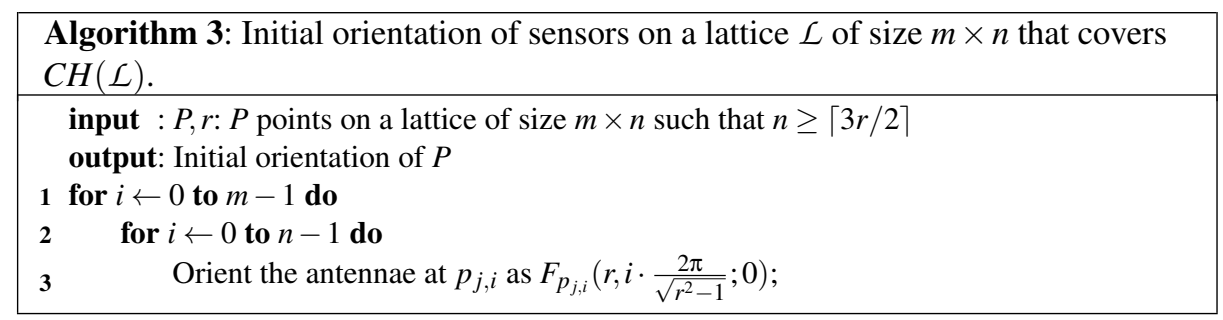

\subsection{Covering the plane}

Next we consider antennae orientation algorithms for covering the entire plane. The case of coverage with two antennae is simple, but coverage with three antennae turns out to be quite intricate and elegant.

Theorem 5. Let $\mathcal{P}$ be the entire plane. We have that $\Phi(2, \mathcal{P})=2 \pi$.

Proof. Assume by contradiction that $\omega:=\Phi(2, \mathcal{P})<2 \pi$; see Figure 5 .

Let $p_{1}, p_{2}$ be two floodlights of angle $\omega$. Assume that there is an initial orientation of $p_{1}$ and $p_{2}$ such that every point in the plane is covered at all times. However, there exists an uncovered wedge $w_{1}$ forming an angle $2 \pi-\omega$ emanating from $p_{1}$ and another uncovered wedge $w_{2}$ forming an angle $2 \pi-\omega$ emanating from $p_{2}$. Clearly, at some time $t$ as the sensors rotate with identical constant speeds the sensor $p_{2}$ will be within the wedge $w_{1}$. But then it is not difficult to see that a planar region is left which is covered by neither $p_{1}$ nor $p_{2}$, which is a contradiction.

Theorem 6. Let $\mathcal{P}$ be the entire plane. We have that $\Phi(3, \mathcal{P})=\pi$. 


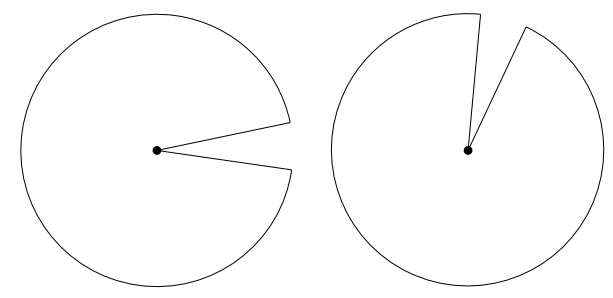

Fig. 6: $\Phi(2, P)=2 \pi$.

Proof. Let $p, q, r$ be three directional sensors in the plane. If the sensors are co-linear then the initial configuration depicted in Figure 6 can be easily seen to be correct.

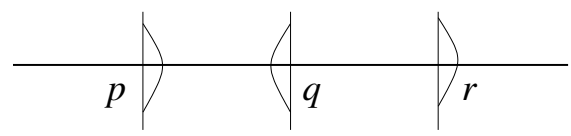

Fig. 7: Initial orientation for three sensors in co-linear position.

Therefore we may assume, without loss of generality, that the three sensors are not in co-linear position. Further we may assume that the line segment $p r$ is horizontal and $q$ is above $p r$. Let $C$ be the circumcircle $C$ of $p, q, r$. Orient $p$ as $F_{p}(l ; 0)$, where $l$ is the tangent of $C$ at $p, q$ as $F_{q}(\pi+\angle(q p r) ; 0)$ and $r$ as $F_{r}(0 ; 0)$ as depicted in Figure $7 \mathrm{a}$. Consider any point $a$ in the circumference of $C$ of pqr. Observe that the angle that each sensor forms with $a$ is equal to the arc; see Figure 7b. Therefore, they intersect at $a$. It can be verified that when $a$ is in the arc $p r, q r$ leave an uncovered wedge with apex at $p$. However, $p$ covers the uncovered wedge. When $a$ is in the arc $r q$, the roles change to $p, q$ and $r$ respectively and when $a$ is in the arc $q p$, the roles change to $p r$ and $q$ respectively. This proves the upper bound if the points are not collinear.

Assume now that $p, q, r$ are collinear. Without loss of generality assume that they are on a horizontal line and the $x$-coordinate of $q$ is greater than the $x$-coordinate of $p$ and smaller than the $x$-coordinate of $r$. Orient $p, q, r$ as $F_{p}(0 ; 0), F_{q}(\pi ; 0)$ and $F_{r}(0 ; 0)$. By Lemma 1, $p$ and $q$ cover the plane at time $t<\pi$ and $q$ and $r$ cover the plane at time $\pi \leq t<2 \pi$.

To prove that the bound is tight, assume by contradiction that $\Phi(3, \mathcal{P})=\pi-\varepsilon$. Assume that at time $t$ the sensors cover the plane. Therefore, there exists a point $a$ in the coverage area of $p$ where two line wedges incident to $q$ and $r$ intersect since two sensor cannot cover the plane as depicted in Figure 7c. However, $a$ is not covered at time $t+\pi$ since $\Phi(3, \mathcal{P})=\pi-\varepsilon$.

Theorem 7. Let $P$ be a set of $n \geq 3$ points in general position and $C H(P)$ be the convex hull on $P$. We have that $\Phi_{r}(P, C H(P)) \geq \pi$ where $r$ is twice the longest edge of the Delaunay Triangulation of $P$. 


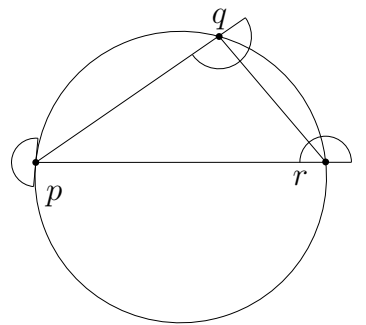

(a) Initial orientation.

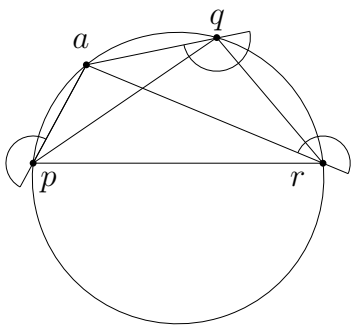

(b) Initial orientation.

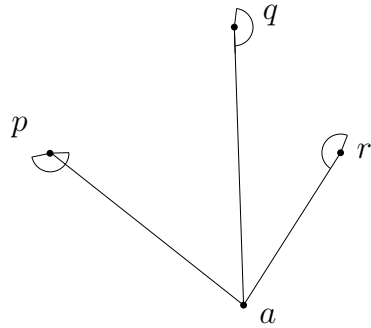

(c) Lower bound.

Fig. 8: Three points covering the plane.

Proof. Consider the Delaunay triangulation $D T(P)$ of $P$. Let $G$ be the dual graph of $D T(P)$ where each triangle $\triangle(u)$ of $D T(P)$ is a vertex $u$ in $G$ and two vertices $u, v$ are adjacent in $G$ if and only if $\triangle(u) \cap \triangle(v) \neq \emptyset$ in $D T(P)$. Observe that unlike Voronoi diagrams, there is no vertex in the dual for the outer face and $G$ is not planar. Let $I$ be a maximal independent set of $G$. For each vertex $u \in I$ we orient the directional sensors that form the triangle $\triangle(u)$ as in Theorem 6. Let $r$ be twice the longest edge of $D T(P)$. We claim that $r$ is always sufficient to cover $C H(P)$. To prove the claim assume on the contrary that it is not sufficient. Therefore, there exists a time where a triangle $\triangle(v)$ is not fully covered. From Theorem $6, v$ is not a neighbor of $u \in I$ since the sensors of $\triangle(u)$ cover all the adjacent triangles at all times. Therefore $I$ is not maximal. This contradicts the assumption.

\section{Coverage with Gap Time at Most $T$}

In this section we study a variant of the problem in which we allow points to be uncovered for a period of time no longer than $T$. Let $\Phi_{r}(P ; \mathcal{R}, T)$ be the infimum over all angles $\phi \leq 2 \pi$ such that if sensors of angle $\phi$ and range $r$ are located at the points then there is an initial orientation of the sensors so that every point is left uncovered for a period of time no longer than $T<2 \pi$. under continuous rotation of the directional sensors. We will prove that in fact the two problems are equivalent.

Theorem 8. $\Phi_{r}(P, \mathcal{R} ; T)=\Phi_{r}(P, \mathcal{R})-T$

Proof. Assume an initial orientation of the sensor in $P$ with angle $\Phi_{r}(P, \mathcal{R})$. For each sensor $p$ of $P$, we will show how to orient $p$ with angle $\Phi_{r}(P, \mathcal{R})-T$ such that every point is uncovered for a period of time no longer than $T$. Let $F_{p}(r, \rho ; 0)$ be the initial orientation of $p$ with angle $\Phi_{r}(P, \mathcal{R})$ such that $\mathcal{R}$ is fully covered at all times. Let the initial orientation of $p$ as $F_{p}(r, \rho+T ; 0)$ with angle $\Phi_{r}(P, \mathcal{R})-T$ We claim that the initial orientation does not leave any point unattained for longer than time $T$. Assume on the contrary that there exists a point $a$ such that it is uncovered for a time greater than 
$T$. Therefore, $a$ is not covered by any sensor $p_{i}$ with angle $\Phi_{r}(P, \mathcal{R})$. This contradicts the assumption.

\section{Software}

We implemented our algorithms in $\mathrm{C}++$ to confirm our results. The programs can be downloaded from http://people.scs.carleton.ca/ omponce/floodlights/index.html.

\section{Conclusion and Open Problems}

We have studied the problem of determining the initial orientation of rotating directional sensors so as to ensure uninterrupted coverage of a planar region under continuous rotation of the antennae. We studied the problem in several settings, including sensors located in lattice and arbitrary configurations as well as for various types of regions. Several open problems remaining concern angle/range tradeoffs. Additional problems concern determining tight bounds on the angle $\Phi(P)$ for arbitrary and specific configurations of points $P$, e.g., points in convex position, etc. In this paper we proved that $\Phi(n, \mathcal{P})$ is equal to $2 \pi$ for $n=2$, and equal to $\pi$ for $n=3$. However, nothing non-trivial is known for $n \geq 4$. Additional interesting questions arise by considering alternative settings concerning the speeds and rotation directions of the antennae, as well as $k$ coverage whereby $k$ antennae are required to monitor all points at all times.

\section{References}

1. M. Cardei and J. Wu. Energy-efficient coverage problems in wireless ad-hoc sensor networks. Computer communications, 29(4):413-420, 2006.

2. V. Chvatal. A combinatorial theorem in plane geometry. Journal of Combinatorial Theory, Series B, 18(1):39-41, 1975.

3. S. Fisk. A short proof of chvátal's watchman theorem. Journal of Combinatorial Theory, Series B, 24(3):374-374, 1978.

4. H. González-Baños. A randomized art-gallery algorithm for sensor placement. In Proceedings of the seventeenth annual symposium on Computational geometry, pages 232-240. ACM, 2001.

5. H. Gupta, S.R. Das, and Q. Gu. Connected sensor cover: self-organization of sensor networks for efficient query execution. In Proceedings of the 4th ACM international symposium on Mobile ad hoc networking \& computing, pages 189-200. ACM, 2003.

6. C.F. Huang and Y.C. Tseng. The coverage problem in a wireless sensor network. Mobile Networks and Applications, 10(4):519-528, 2005.

7. D. Lee and A. Lin. Computational complexity of art gallery problems. Information Theory, IEEE Transactions on, 32(2):276-282, 1986.

8. S. Meguerdichian, F. Koushanfar, M. Potkonjak, and M.B. Srivastava. Coverage problems in wireless ad-hoc sensor networks. In INFOCOM 2001. Twentieth Annual Joint Conference of the IEEE Computer and Communications Societies. Proceedings. IEEE, volume 3, pages 1380-1387. IEEE, 2001. 
9. J. O'Rourke. Art gallery theorems and algorithms, volume 57. Oxford University Press Oxford, 1987.

10. S. Poduri and G.S. Sukhatme. Constrained coverage for mobile sensor networks. In Robotics and Automation, 2004. Proceedings. ICRA'04. 2004 IEEE International Conference on, volume 1, pages 165-171. IEEE, 2004.

11. J. Urrutia. Art gallery and illumination problems. Handbook of computational geometry, pages 973-1027, 2000.

12. X. Wang, G. Xing, Y. Zhang, C. Lu, R. Pless, and C. Gill. Integrated coverage and connectivity configuration in wireless sensor networks. In Proceedings of the 1st international conference on Embedded networked sensor systems, pages 28-39. ACM, 2003. 